\title{
Metric-Based Visual Acuity and Defocus Curve Simulation of Two Multifocal Intraocular Lens Models
}

This article was published in the following Dove Press journal:

Clinical Ophthalmology

\author{
Lin $\mathrm{He}^{1}$ \\ Xin Hong ${ }^{2}$ \\ Rajaraman Suryakumar ${ }^{2}$ \\ Ramesh Sarangapani ${ }^{2}$ \\ 'Department of Pathology, University of \\ Texas Southwestern Medical Center, \\ Dallas, TX, USA; ${ }^{2}$ Alcon Research LLC. \\ Fort Worth, TX, USA
}

Purpose: To predict clinical defocus curve performance of the PanOptix intraocular lens (IOL) model TFNT00, a population-based image quality metric was applied to a pseudophakic eye model.

Methods: Visual acuity (VA) was simulated using a 2-surface reduced eye model. For each virtual eye, the derived corneal surface was combined with scaled IOL surface. Corneal power and aberration, anterior chamber depth, and pupil size were iterated using a Monte-Carlo approach. Image quality of the IOLs was assessed using the total aberration map to compute the amplitude point spread function. A diffraction-normalized light-in-the-bucket metric was calculated for each virtual eye for defocuses from $-3.5 \mathrm{D}$ to $+1.0 \mathrm{D}$ (step size $0.25 \mathrm{D}$ ) and transformed to VAs and defocus curves. Simulated VA for the ReSTOR +3.0 D lens was used to generate a calibration function by linear regression correlation of simulated data with clinical VA data. Simulated TFNT00 VA was then validated by comparing defocus curves to clinical TFNT00 data.

Results: From $-3.5 \mathrm{D}$ to $+1.0 \mathrm{D}$, the simulated defocus curve was generally consistent with the defocus curve from the TFNT00 clinical trial. The mean absolute difference was $0.022 \log$ MAR ( $\sim 1$ letter) for simulated VA versus clinical trial VA.

Conclusion: IOL image quality can be assessed using a population-based virtual eye model to simulate VA and predict clinical performance. Computational modeling and simulation can be applied to future IOL development before clinical trials are conducted.

Keywords: light-in-the-bucket, modulation transfer function, pseudophakic eye model

\section{Plain Language Summary}

Simulation studies can evaluate the effectiveness of ophthalmic devices without the risks to patients, such as surgical complications or undesired visual outcomes. The goal of the present study was to develop a simulation method to predict performance of a trifocal intraocular lens (IOL). A cohort of 1000 virtual eyes was generated and a population-based image quality metric was applied to a virtual pseudophakic eye model to predict clinical performance. After simulated visual acuity experiments, visual acuity was assessed in cataract patients with a trifocal IOL, 6 months after surgery. The results of that clinical assessment served as validation in a comparison between simulated and clinical visual outcomes. Predicted IOL performance correlated well with clinical results, suggesting the simulation method may be applied to future IOL development. These analytical techniques may help optimize product design and develop performance targets for clinical trials.

\section{Introduction}

Department of Pathology, University of Texas Southwestern Medical Center, 5323 Harry Hines Blvd, Dallas, TX 75390, USA Tel $+|\mathrm{I}-8| \mathrm{I}-302-5666$

Email retinoblastoma|3q14@gmail.com
Multifocal intraocular lenses (IOLs) are a significant innovation in cataract surgery, mitigating the effects of presbyopia by increasing spectacle independence compared with standard monofocal lenses. ${ }^{1,2}$ Typically, these IOLs are designed with 2 focal 
points (bifocal) that provide the necessary optical power to focus incoming light and provide the patient with optimal distance and near vision. However, many daily tasks, such as computer work, are performed at an intermediate distance. Intermediate distance ranges from 60 to $80 \mathrm{~cm}$, with $60 \mathrm{~cm}$ considered to be functionally preferred by most people., ${ }^{3,4}$

In contrast to bifocal lenses, trifocal lenses create 3 focal points so that optimal vision is achieved at near, intermediate, and distance.$^{5-7}$ Optical bench studies have investigated the effect of a third focal point on the quality of vision at various distances; a study of 2 commercially available trifocal IOLs, the AT LISA tri 839MP (Carl Zeiss Meditec, Dublin, CA, USA) and the PhysIOL FineVision MICRO F12 (PhysIOL, Liège, Belgium), demonstrated improved intermediate visual acuity (VA) at $80 \mathrm{~cm}$ compared with bifocal IOLs. ${ }^{8}$ A new trifocal IOL, the AcrySof ${ }^{\circledR}$ IQ PanOptix ${ }^{\circledR}$ Presbyopia-Correcting IOL model TFNT00 (Alcon Vision LLC, Fort Worth, TX), is designed to provide $20 / 25$ vision or better at $40 \mathrm{~cm}, 60 \mathrm{~cm}$, and distance. ${ }^{9}$ For near and intermediate vision, TFNT00 provides a continuous range of vision from 40 to $80 \mathrm{~cm}$, with an intermediate add power at $60 \mathrm{~cm}$ that has been determined to be suitable for everyday activities like computer work for most viewers. ${ }^{4,10,11}$

To fully understand the performance of multifocal IOLs, clinicians often use empirical methods to assess vision such as the defocus curve. In this assessment, ophthalmic lenses of various optical powers are placed in front of the eye and VA is measured. The change in VA as a function of lens power demonstrates the level of vision that is maintained over all distances measured. Although visual performance measurements are an optimal way of assessing the safety and performance of an IOL, there are several challenges to clinical testing. In addition to the risks associated with surgical interventions such as IOL implantation, clinical trials require many months of follow-up to demonstrate lens performance. Alternatively, IOL performance can be assessed using computational modeling and simulation techniques, providing crucial information on lens effectiveness before clinical trials are conducted. ${ }^{12-14}$ Furthermore, when conducted as a population-based simulation, these analytical techniques may help in optimization of product design, provide guidance on performance targets for clinical trials, and aid in selecting appropriate clinical endpoints. ${ }^{15}$ Pseudophakic eye models have been developed to measure the performance of monofocal and bifocal IOLs that simulate the retinal image quality expected from the different diffractive-refractive optical elements. ${ }^{13}$ Results of such modeling experiments have demonstrated close agreement with clinical optical performance and thus provide a method of predicting the image quality that may be achieved after IOL implantation. ${ }^{13}$

This report describes the validation of an eye model to simulate and predict the clinical performance of TFNT00 using well-established image quality metrics. ${ }^{12-16}$ Previously, an optical bench evaluation comparing TFNT00 to the AcrySof ${ }^{\circledR}$ IQ ReSTOR ${ }^{\circledR}+3.0$ D multifocal IOL (model SN6AD1; Alcon) showed that TFNT00 provided equivalent near and distance performance and improved intermediate performance. ${ }^{11}$ The goal of the present study was to develop a population-based image quality metric, calibrated with known existing clinical data for SN6AD1, and apply to a pseudophakic eye model to predict the clinical defocus curve performance of the TFNT00 IOL.

\section{Methods}

\section{Intraocular Lenses}

SN6AD1 and TFNT00 are diffractive IOLs with aspheric corrections for a corneal spherical aberration of $0.1 \mu \mathrm{m}$ at 6-mm pupil size. SN6AD1 is an apodized bifocal IOL with a diffractive zone of $3.6 \mathrm{~mm}$ that has active diffraction orders of zeroth and first powers, creating a near add power of +3.00 D. TFNT00 is a nonapodized trifocal IOL with a diffractive zone of $4.5 \mathrm{~mm}$ that creates near and intermediate add powers of $+3.25 \mathrm{D}$ and $+2.17 \mathrm{D}$, respectively. ${ }^{17}$ It has active diffractive orders of the zeroth, second, and third magnitude that create distance, intermediate, and near focal points, respectively.

\section{Simulation Model}

Visual acuity was simulated for SN6AD1 and TFNT00 using a 2-surface reduced eye model implemented using MATLAB (MathWorks, Inc., Natick, MA, USA) programming language. The development of this model for multifocal IOLs in pseudophakic eyes was previously reported. ${ }^{13}$ Briefly, the wavefront aberrations of the pseudophakic eye were computed as the sum of the stimulus vergence, the diffractive surface of the IOL, and the refractive elements (ie, the corneal surface and the refractive surface of the IOL). ${ }^{13}$ The corneal surface was reconstructed using clinical data from pupil diameter, anterior chamber depth, corneal power, and high-order corneal aberration from literature (Figure 1). ${ }^{18-20}$ The wavefront aberrations for the stimulus and the refractive elements were defined at the corneal plane. ${ }^{20}$ The wavefront aberration of the 


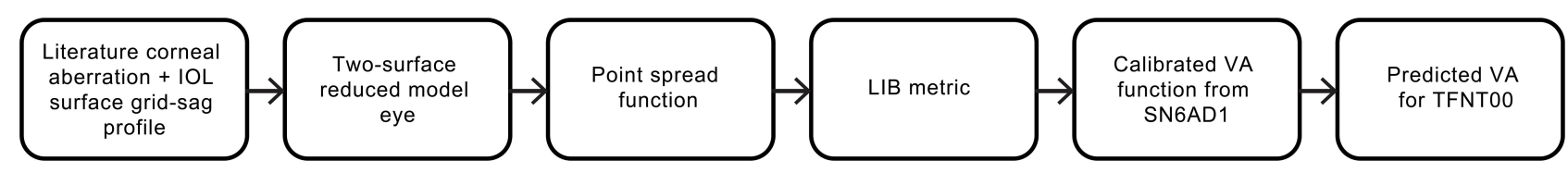

Figure I Flow chart for metric-based visual acuity simulation. IOL, intraocular lens; LIB, light-in-the-bucket; VA, visual acuity.

diffractive optical element, defined at the IOL plane, was modeled as a finite thickness kinoform lens based on the formula for phase function. The IOL surface model was extracted from a theoretical design of the IOL surface using a Zemax grid-sag profile (Zemax LLC, Kirkland, WA, USA) that defined base surface perturbations and subtracted base curvature. Both surfaces were scaled and combined at the corneal plane where defocus was defined. A scaling magnification factor was incorporated to account for the shift in IOL grid-sag profile from the IOL plane to the entrance pupil plane, which is dependent on the corneal power, anterior chamber depth, and refractive indices of both the aqueous humor and the IOL material.

A cohort of 1000 virtual eyes was generated by sampling model parameters (Table 1) for pupil diameter, anterior chamber depth, corneal power, and individual anterior corneal high-order aberration. ${ }^{20}$ For each virtual eye, the derived corneal surface was combined with scaled IOL surface. Corneal power and aberration, anterior chamber depth, and pupil size were iterated using a Monte-Carlo approach. Image quality of the IOLs was assessed using the total aberration map to compute the amplitude point spread function, the Fourier transform of the pupil function associated with the phase-delay map of the diffractive optical element at the pupil plane. $^{13}$ A diffraction-normalized light-in-the-bucket (LIB) metric was then used to quantify the total amount of light in the central core of the point spread function (Equation 1). ${ }^{14}$ LIB was selected based on a previous study, in which the Monte-Carlo approach was applied to both modulation transfer function (MTF) area and LIB metrics. LIB had better

Table I Parameters and Corresponding Values Used in the 2-Surface Reduced Eye Model

\begin{tabular}{|l|l|}
\hline Parameter & Value \\
\hline Wavelength, $\mathrm{nm}$ & 550 \\
Refractive index of aqueous humor, $\mathrm{n}_{\mathrm{aq}}$ & $\mathrm{I} .336$ \\
Refractive index of IOL, $\mathrm{n}_{\mathrm{IOL}}$ & $\mathrm{I} .55 \mathrm{I}$ \\
Mean $\pm \mathrm{SD}$ pupil diameter, mm & $3.50 \pm 0.80$ \\
Mean $\pm \mathrm{SD}$ anterior chamber depth, $\mathrm{mm}$ & $4.60 \pm 0.30$ \\
Mean $\pm \mathrm{SD}$ corneal power, D & $43.4 \pm 2.2$ \\
\hline
\end{tabular}

Abbreviation: IOL, intraocular lens. correlation with clinical VA than MTF area $\left(\mathrm{R}^{2}=0.92\right.$ vs 0.65 , respectively). ${ }^{15}$

$$
\mathrm{LIB}=\int_{\mathrm{DLcore}} \operatorname{PSF}_{\mathrm{N}}(\mathrm{x}, \mathrm{y}) d x d y
$$

In this equation, $\mathrm{PSF}_{\mathrm{N}}$ is the normalized point spread function. The domain of integration is the central core of a diffraction-limited PSF for the same pupil size. The LIB metric was calculated for each virtual eye for defocuses from $-3.5 \mathrm{D}$ to $+1.0 \mathrm{D}$, with a step size of $0.25 \mathrm{D} .^{21}$

The simulated VA from the SN6AD1 lens was used to generate a calibration function by correlating simulated data with clinical VA data for SN6AD1 using a linear regression model. ${ }^{12,16}$ The calibration function was applied to the clinical VA data of the other IOLs of interest (such as the TFNT00 lens in the study) to transform the LIB metric to simulated VAs and defocus curves. SN6AD1 clinical study results were used as the training dataset to build the linear regression model, while the ultimate TFNT00 clinical study results were used as the validation dataset. Simulated and clinical VA was assessed at distance $(4 \mathrm{~m}), 80 \mathrm{~cm}(-1.25 \mathrm{D})$, $60 \mathrm{~cm}(-1.67 \mathrm{D})$, and $40 \mathrm{~cm}(-2.5 \mathrm{D})$, which were either measured or linearly interpolated from the defocus curve. The relationship of the LIB metric for SN6AD1 (estimated in virtual eyes) and clinical binocular VA for the SN6AD1 lens (defocus $-3.5 \mathrm{D}$ to $+1.0 \mathrm{D}$ ) was determined using multiple models (linear, exponential, logit); each model demonstrated comparable goodness-of-fit. ${ }^{21}$ Consequently, the linear calibration function was used to simulate VA for TFNT00 (Equation 2) in a population-based model of 100 virtual eyes:

$$
\text { Binocular } V A=-0.238 * \log (L I B)-0.200
$$

For the calibration function, the LIB metric was derived from monocular condition and the clinical VA was representative of clinical binocular measurement. Therefore, the binocular summation factor was incorporated and assumed to be constant at different defocus and can be addressed from the linear calibration function.

\section{Clinical Validation}

After the simulated VA experiments were conducted, a 1-year, prospective, multicenter, single-arm, nonrandomized clinical 
trial of subjects implanted bilaterally with TFNT00 was initiated (ClinicalTrials.gov identifier, NCT02529488). ${ }^{9}$ The results of that clinical trial served as validation in a head-tohead comparison between simulated and clinical visual outcomes. The clinical study was conducted in accordance with the principles of the Declaration of Helsinki, and in compliance with Good Clinical Practice, ISO 14155:2011. Approvals were obtained from relevant Institutional Review Boards or Ethics Committees (Bellberry Human Research Ethics Committee; Ethik-Kommission des Fachbereichs Medizin der Johann Wolfgang Goethe-Universität; Ethikkommission der Med. Fakultät Heidelberg; Les Comités de Protection des Personnes Ouest VI; Comitato etico per la Sperimentazione Clinica delle Province di Verona e Rovigo; Comité Ético de Inv. Clínica de Asturias; De medisch-ethische toetsingscommissie van het azM en Maastricht University; Comité ético de investigación clínica de Euskadi; Comité de Ética de la Universidad de Chile; Servicio de Salud Metropolitano Oriente; London Harrow Research Ethics Committee; Marina Médica; Azienda Ospedaliero Univ Careggi; OLV Ziekenhuis vzw). Subjects provided informed consent before any study-related procedures were initiated. Binocular defocus testing was performed under photopic conditions $\left(\sim 85 \mathrm{~cd} / \mathrm{m}^{2}\right)$ with a $100 \%$ contrast Early Treatment Diabetic Retinopathy Study chart at $4 \mathrm{~m}$. Subjects were defocused from manifest refraction using $-5.00 \mathrm{D}$ and $+2.00 \mathrm{D}$ spherical corrections by decreasing minus or plus power in $0.5-\mathrm{D}$ increments until only the best distance correction remained; VA was recorded in $\log$ MAR.

\section{Results}

\section{Two-Surface Model Eye Qualification}

Per the International Organization for Standardization 2014, the through-focus MTF was required testing for multifocal IOLs. To qualify the 2-surface reduced model eye output, the through-focus MTF at 50 line pair/mm was calculated using the reduced eye model for TFNT00 with matched cornea, spherical aberration of $0.10 \mu \mathrm{m}$, and compared with the MTF simulated for the Alcon-Navarro eye model in Zemax (Figure 2). In this way, MTFs were generated from the 2 independent eye models and software algorithms. For a bifocal IOL, the 2-surface eye model produced through-focus MTF comparable to a more complicated Alcon-Navarro eye model. ${ }^{22}$

\section{Simulated Prediction of Visual Acuity for TFNT00}

The simulated and clinical comparison of binocular VA for SN6AD1 is shown in Figure 3. The simulated and clinically observed binocular VA for the TFNT00 and SN6AD1 IOLs are summarized in Table 2. At the intermediate distance of $60 \mathrm{~cm}$, the simulated VA for TFNT00 was $0.054 \operatorname{logMAR}$ compared with $0.134 \log$ MAR for SN6AD1. Values at $60 \mathrm{~cm}(-1.67 \mathrm{D})$ were linearly interpolated from values at $67 \mathrm{~cm}(-1.5 \mathrm{D})$ and $50 \mathrm{~cm}(-2.0$ D). The simulated VA for TFNT00 at every defocus was better than $0.1 \log$ MAR, demonstrating improved performance at intermediate distances compared with the simulated VA for the SN6AD1. The predicted difference in

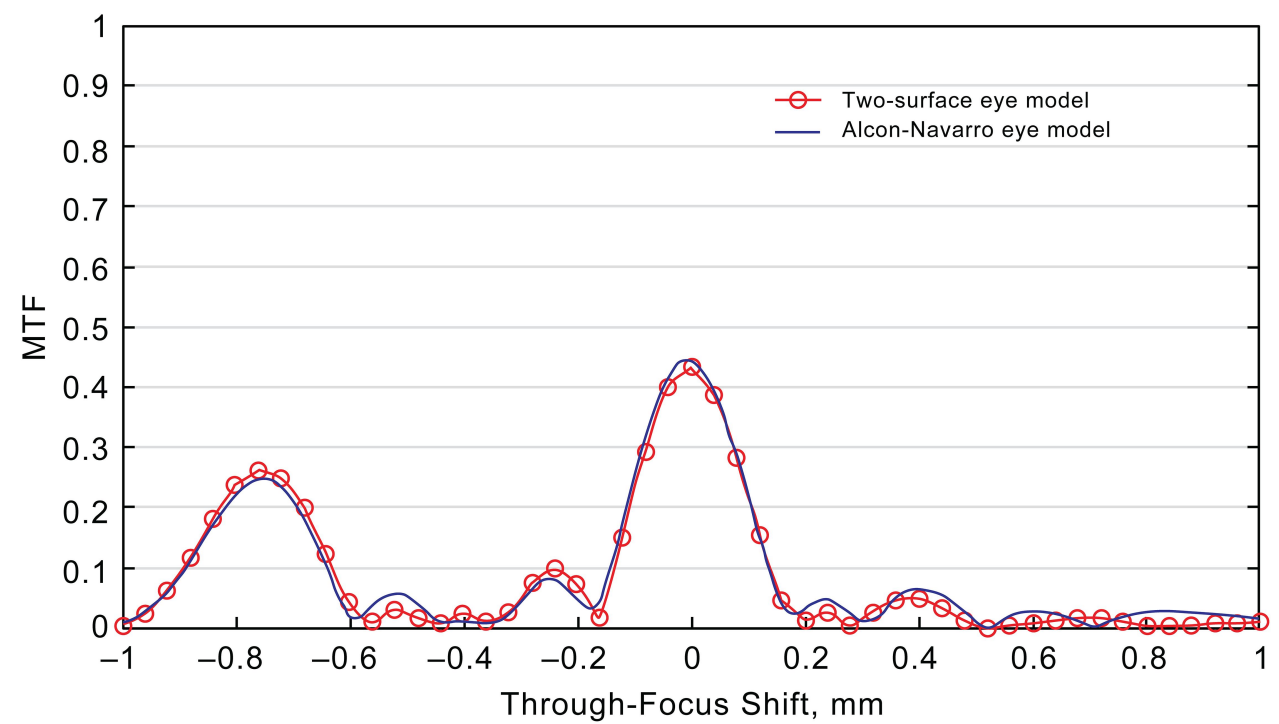

Figure 2 Through-focus MTF at 50 line pair/mm generated by 2-surface eye model (red open circles, red line) compared with Alcon-Navarro eye model (blue line). MTF, modulation transfer function. 


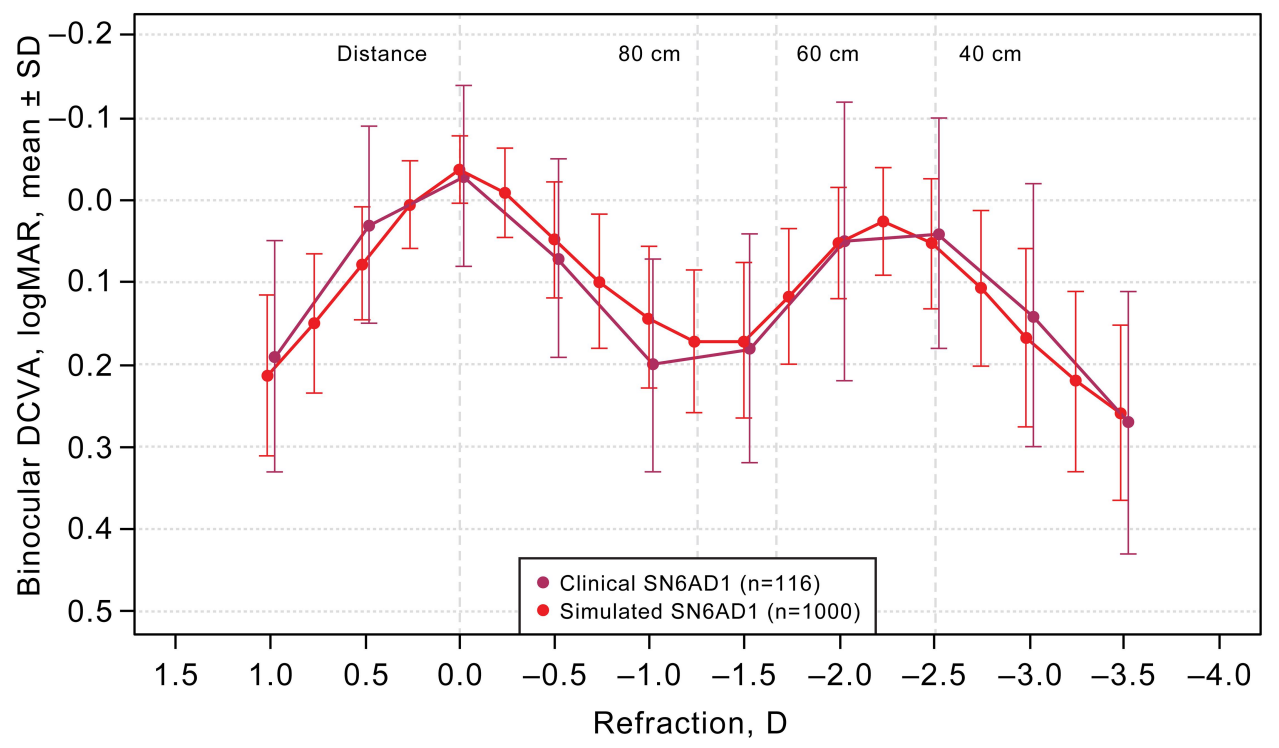

Figure 3 Simulated defocus curve vs clinical defocus curve of SN6ADI. Data on File. Alcon Laboratories, Fort Worth, TX. Abbreviation: DCVA, distance-corrected visual acuity.

binocular VA between SN6AD1 and TFNT00 was 0.076 $\log \mathrm{MAR}$ at $80 \mathrm{~cm}$ and $0.080 \log \mathrm{MAR}$ at $60 \mathrm{~cm}$.

\section{Comparison of Simulated TFNT00 Defocus Curves with Clinical TFNT00 Defocus Curves}

Clinical defocus curves were measured 6 months postoperatively in a clinical trial in which TFNT00 was implanted bilaterally in 149 subjects. ${ }^{9}$ The clinical defocus curve generated from this trial was compared with the TFNT00 defocus curve generated from the cohort of 100 simulated eyes. From $-3.5 \mathrm{D}$ to $+1.0 \mathrm{D}$, the simulated defocus curve was generally consistent with the defocus curve from the clinical trial (Figure 4). ${ }^{15}$ The largest difference between the simulated VA and clinical VA was $0.059 \log$ AAR ( $<3$ letters) at $-3.0 \mathrm{D}$.

Table 2 Summary of Clinical and Simulated Binocular Visual Acuity at $4 \mathrm{~m}$ (Distance) and at $80 \mathrm{~cm}, 60 \mathrm{~cm}$, and $40 \mathrm{~cm}$

\begin{tabular}{|l|l|l|l|l|}
\hline & $\begin{array}{l}\text { TFNT00, } \\
\text { Simulated }\end{array}$ & $\begin{array}{l}\text { TFNT00, } \\
\text { Clinical }\end{array}$ & $\begin{array}{l}\text { SN6ADI, } \\
\text { Simulated }\end{array}$ & $\begin{array}{l}\text { SN6AD I, } \\
\text { Clinical }^{\text {b }}\end{array}$ \\
\hline $\begin{array}{l}\text { Iteration/ } \\
\text { sample size }\end{array}$ & 1000 & 134 & 1000 & 116 \\
$4 \mathrm{~m}$ & -0.027 & -0.037 & -0.039 & -0.030 \\
$80 \mathrm{~cm}^{\text {a }}$ & 0.095 & 0.083 & 0.171 & 0.190 \\
$60 \mathrm{~cm}^{\mathrm{a}}$ & 0.054 & 0.058 & 0.134 & 0.137 \\
$40 \mathrm{~cm}$ & 0.096 & 0.066 & 0.052 & 0.040 \\
\hline
\end{tabular}

Notes: a Note that values at $60 \mathrm{~cm}(-1.67 \mathrm{D})$ were linearly interpolated from clinical and simulated visual acuity at $67 \mathrm{~cm}(-1.5 \mathrm{D})$ and $50 \mathrm{~cm}(-2.0 \mathrm{D})$; values at $80 \mathrm{~cm}(-1.25 \mathrm{D})$ were linearly interpolated from $-1.0 \mathrm{D}$ and $-1.5 \mathrm{D}$. ${ }^{\mathrm{b}} \mathrm{Data}$ on File. Alcon Laboratories, Fort Worth, TX.
The mean absolute difference between simulated and clinical defocus curves was $0.022 \operatorname{logMAR}(\sim 1$ letter) for the simulated VA compared with the clinical trial VA. Between -2.5 D $(40 \mathrm{~cm})$ and $0 \mathrm{D}$ (distance), simulated and clinical VAs were all better than $0.1 \log$ MAR (Snellen 20/25), indicating highly functional visual performance of the TFNT00 IOL.

\section{Discussion}

Simulated and virtual clinical trials allow effectiveness of ophthalmic devices such as IOLs to be evaluated without the risks to human subjects associated with clinical trials, such as surgical complications, long periods of follow-up for patients, or undesired visual outcomes. In this study, we have developed a novel method of predicting clinical performance of the ReSTOR +3.0 D IOL (SN6AD1) using a pseudophakic eye model. This approach was then applied to the PanOptix IOL (TFNT00), and the simulated results showed that the design intent of the IOL was met in terms of clinically significant improvement in intermediate and near vision. After the simulation results were obtained, a clinical trial of subjects who received TFNT00 was conducted, and the results were compared with the simulated results as the final validation step. The model predictions using the LIB metric for TFNT00 showed improved performance compared with the SN6AD1 for intermediate vision (60 and $80 \mathrm{~cm}$ ), with approximately 1 line of improvement at $80 \mathrm{~cm}$ and comparable performance for near $(40 \mathrm{~cm})$ and distance $(4 \mathrm{~m})$ vision. These results generally agreed with previously reported standard bench measurements. ${ }^{11}$ 


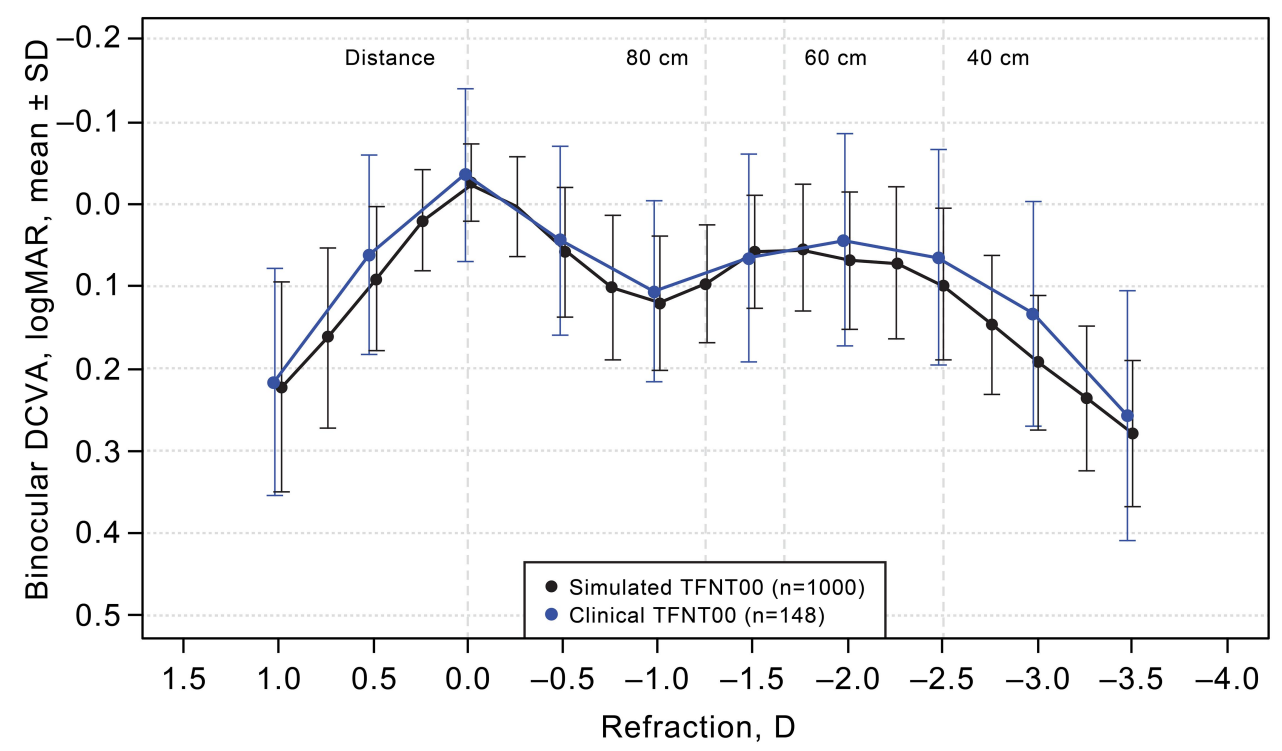

Figure 4 Simulated defocus curve of TFNT00 vs clinical defocus curve from a clinical trial. ${ }^{9}$ DCVA, distance-corrected visual acuity.

The simulation approach used in the present study has advantages over optical bench performance studies. For example, TFNT00 binocular VA was simulated by correlating the LIB metric with the clinical binocular VA of SN6AD1, unlike in a previous study of trifocal IOLs that used Badal optometer images and did not take clinical binocular effects into account. ${ }^{23}$ The validity of the model was confirmed by comparing simulated results with data from clinical trials of subjects who received TFNT00 after cataract removal. The subjects in these studies were representative of typical subjects who desire the ability to perform daily tasks at intermediate distances, such as reading a computer screen, ${ }^{24}$ without the use of corrective spectacles. Optical bench imaging studies are often performed in an ideal aberration-free or aberration-matched condition, but may not fully represent the real-world visual experience and cannot reflect variations in ocular biometry among human subjects. ${ }^{11}$ The results of this study showed that the differences between simulated and clinical VA were small, indicating that this population-based simulation of defocus curve can serve as a useful tool for optimizing IOL design. There was some variance present in the clinical VA outcomes for both IOLs that may have been introduced by the calibration function. The LIB metric was calculated based on monocular condition whereas the clinical defocus curves were measured with both eyes best corrected, which could have resulted in slight anisotropy. Additionally, in another pilot study of 20 subjects implanted with TFNT00, the binocular defocus curve demonstrated comparable performance at intermediate and far distances and improved performance for near vision compared with 14 subjects implanted with the TECNIS Symfony ${ }^{\circledR}$ extended range of vision IOL (Johnson \& Johnson Vision, Santa Ana, CA, USA). ${ }^{7}$ The reported mean \pm SD distance-corrected intermediate VA at $60 \mathrm{~cm}$ was $0.06 \pm 0.10 \log$ MAR for subjects implanted with TFNT00, which agrees with the simulated VA for TFNT00 obtained in this study, indicating that the simulated eye model is useful for predicting visual performance. ${ }^{7}$

Many image quality metrics have been investigated in previous studies, ${ }^{14,16}$ and a recent study using an MTF-based metric to predict VA of pseudophakic eyes demonstrated good predictability. ${ }^{25}$ In a study comparing the predictability of MTFa and LIB, both metrics demonstrated a strong correlation with clinical VA (MTFa, $\left.\mathrm{R}^{2}=0.94 ; \mathrm{LIB}, \mathrm{R}^{2}=0.90\right) .{ }^{26}$ However, the predictability of MTFa diminished more rapidly than LIB when increasing pupil size, indicating its sensitivity to pupil dependent aberrations. Further studies are needed to determine whether other optical parameters may have a similar effect on MTFa. These findings support the approach used in the present study for use of the LIB metric to predict clinical performance.

There were several limitations of the simulation-based model used in this study. The model assumed ideal IOL alignment, and several sources of potential error were not accounted for, including post-operative IOL decentration or tilt and corneal incision. The LIB metric was calibrated assuming monochromatic conditions at a wavelength of $550 \mathrm{~nm}$, and, therefore, polychromatic factors were not evaluated in the analysis. Moreover, the apodized 
(SN6AD1) and nonapodized (TFNT00) design of the IOLs may have introduced bias toward the distance and near diffraction images, respectively. ${ }^{13}$ Because the prediction method uses a calibration curve generated using data obtained under particular clinical conditions, actual clinical results may vary if clinical conditions are different. Furthermore, metrics that have been shown to be predictive of clinical outcome (eg, the visual Strehl ratio and the image quality metric) were also not included in the analysis. The calibration function to determine the relationship between LIB metric and VA was estimated using a linear regression model; alternative nonlinear models might be used to derive the calibration function. However, the goodness of fit of the linear model may suggest that the benefit of nonlinear models may be modest at best. Overall, the LIB metric provided good correlation with clinical binocular VA and therefore served as a useful indicator to simulate VA. Lastly, the error limit of the parameters of Equation 2, which was used to simulate VA for TFNT00, was not determined.

In summary, the novel simulation method used to predict the visual performance of the TFNT00 trifocal IOL agreed well with previously reported optical bench evaluations and clinical data. The predicted lens performance correlated well with actual clinical results, suggesting that the simulation method may be a useful modeling tool for clinical research. The metric-based method used in this study can be used to simulate clinical performance for other trifocal models for which the surface profile has been determined. Therefore, this work provides a foundation for future simulation studies of trifocal lenses, which will address the limitations of the current model to provide a more realistic clinical trial simulation for TFNT00 and other IOL models.

\section{Abbreviations}

D, diopter; DCVA, distance-corrected visual acuity; IOL, intraocular lens; LIB, light-in-the-bucket; logMAR, logarithm of the minimum angle of resolution; MTF, modulation transfer function; VA, visual acuity.

\section{Acknowledgments}

The authors wish to thank Myoung Choi, Srichand Jasti (Alcon employees), Yueai Liu (former Alcon employee), and Arthur Bradley (Indiana University) for their contributions. Aldo Martinez of Alcon Vision LLC provided critical review of the manuscript. Medical writing support was provided by Catherine DeBrosse, PhD, of ICON (North Wales, PA), and was funded by Alcon Research LLC (Fort Worth, TX). Presented in part at the 2018 American Society of Cataract and Refractive Surgery Annual Meeting, April 13-17, 2018, Washington, DC, at the 2017 Association for Research in Vision and Ophthalmology, May 7-11, 2017, Baltimore, MD, and at the 2018 Association for Research in Vision and Ophthalmology, April 29-May 3, 2018, Honolulu, HI.

\section{Funding}

This work was sponsored by Alcon Research LLC, Fort Worth, TX, USA.

\section{Disclosure}

Xin Hong, Rajaraman Suryakumar, and Ramesh Sarangapani are employees of Alcon. Lin He was an Alcon employee at the time of the study. The authors report no other potential conflicts of interest for this work.

\section{References}

1. Nijkamp MD, Dolders MG, de Brabander J, et al. Effectiveness of multifocal intraocular lenses to correct presbyopia after cataract surgery: a randomized controlled trial. Ophthalmology. 2004;111(10):1832-1839. doi:10.1016/j.ophtha.2004.05.023

2. Calladine D, Evans JR, Shah S, Leyland M. Multifocal versus monofocal intraocular lenses after cataract extraction. Cochrane Database Syst Rev. 2012;9:CD003169.

3. Working Safely with Video Display Terminals. Washington, DC: Occupational Safety and Health Administration, US Department of Labor; 1997.

4. American Optometric Association. The effects of computer use on eye health and vision. Available from: https:/www.aoa.org/Documents/opto metrists/effects-of-computer-use.pdf. Accessed April 2, 2019.

5. Gatinel D, Pagnoulle C, Houbrechts Y, Gobin L. Design and qualification of a diffractive trifocal optical profile for intraocular lenses. J Cataract Refract Surg. 2011;37(11):2060-2067. doi:10.1016/j. jcrs.2011.05.047

6. Marques EF, Ferreira TB. Comparison of visual outcomes of 2 diffractive trifocal intraocular lenses. J Cataract Refract Surg. 2015;41(2):354-363. doi:10.1016/j.jcrs.2014.05.048

7. Ruiz-Mesa R, Abengozar-Vela A, Ruiz-Santos M. A comparative study of the visual outcomes between a new trifocal and an extended depth of focus intraocular lens. Eur J Ophthalmol. 2018;28 (2):182-187. doi:10.5301/ejo.5001029

8. Carson D, Hill WE, Hong X, Karakelle M. Optical bench performance of AcrySof $^{\circledR}$ IQ ReSTOR ${ }^{\circledR}$, AT LISA ${ }^{\circledR}$ tri, and FineVision ${ }^{\circledR}$ intraocular lenses. Clin Ophthalmol. 2014;8:2105-2113.

9. Bala C, Martinez AA, Kohnen T Multicenter visual outcomes evaluation of a new trifocal presbyopia correcting IOL - 12 month results. Presented at: American Society of Cataract and Refractive Surgery, April 13-17, 2018; Washington, DC.

10. Kohnen T. First implantation of a diffractive quadrafocal (trifocal) intraocular lens. J Cataract Refract Surg. 2015;41(10):2330-2332. doi:10.1016/j.jcrs.2015.11.012

11. Lee S, Choi M, Xu Z, et al. Optical bench performance of a novel trifocal intraocular lens compared with a multifocal intraocular lens. Clin Ophthalmol. 2016;10:1031-1038. doi:10.2147/OPTH. S106646

12. Marsack JD, Thibos LN, Applegate RA. Metrics of optical quality derived from wave aberrations predict visual performance. $J$ Vis. 2004;4(4):322-328. doi:10.1167/4.4.8 
13. Ravikumar S, Bradley A, Thibos LN. Chromatic aberration and polychromatic image quality with diffractive multifocal intraocular lenses. J Cataract Refract Surg. 2014;40(7):1192-1204. doi:10.1016/ j.jers.2013.11.035

14. Thibos LN, Hong X, Bradley A, Applegate RA. Accuracy and precision of objective refraction from wavefront aberrations. $J$ Vis. 2004;4(4):329-351. doi:10.1167/4.4.9

15. He J, Hong X, Sarangapani R Population-based simulation using image quality metrics to predict visual acuity in pseudophakic patients implanted with trifocal IOLs [poster 4209]. Presented at: Association for Research in Vision and Ophthalmology, May 7-11, 2017; Baltimore, MD.

16. Cheng X, Bradley A, Hong X, Thibos LN. Relationship between refractive error and monochromatic aberrations of the eye. Optom Vis Sci. 2003;80(1):43-49. doi:10.1097/00006324-20030100000007

17. Alcon Vision LLC. AcrySof IQ PanOptix Product Information. Available from: https://kategorizacia.mzsr.sk/Pomocky/Download/ CommentAttachment/7699. Accessed June 21, 2019.

18. Jasti S. Data on File. Fort Worth, TX: Alcon Research, Ltd; 2012.

19. Badeaux K, Bolar L, Seaman J, Knighten S. Diagnostic Study of Intraocular Lens Outcomes. Fort Worth, TX: Alcon Research, Ltd; 2013.
20. Wang L, Koch DD. Ocular higher-order aberrations in individuals screened for refractive surgery. J Cataract Refract Surg. 2003;29 (10):1896-1903. doi:10.1016/S0886-3350(03)00643-6

21. He J, Jasti S, Suryakumar R, Sarangapani R Comparison between simulated and clinical visual performance in pseudophakic patients with trifocal intraocular lens [abstract]. Presented at: Association for Research in Vision and Ophthalmology, April 29-May 3, 2018; Honolulu, Hawaii.

22. Hong X. Data on File. Fort Worth, TX: Alcon Research, Ltd; 2003.

23. Carson D, Xu Z, Alexander E, et al. Optical bench performance of 3 trifocal intraocular lenses. J Cataract Refract Surg. 2016;42 (9):1361-1367. doi:10.1016/j.jcrs.2016.06.036

24. Kohnen T, Herzog M, Hemkeppler E, et al. Visual performance of a quadrifocal (trifocal) intraocular lens following removal of the crystalline lens. Am J Ophthalmol. 2017;184:52-62. doi:10.1016/j. ajo.2017.09.016

25. Alarcon A, Canovas C, Rosen R, et al. Preclinical metrics to predict through-focus visual acuity for pseudophakic patients. Biomed $\mathrm{Opt}$ Express. 2016;7(5):1877-1888. doi:10.1364/BOE.7.001877

26. Armengol J, Garzon N, Vega F, Altemir I, Millan MS. Equivalence of two optical quality metrics to predict the visual acuity of multifocal pseudophakic patients. Biomed Opt Express. 2020;11(5):2818-2829. doi:10.1364/BOE.388531
Clinical Ophthalmology

\section{Publish your work in this journal}

Clinical Ophthalmology is an international, peer-reviewed journal covering all subspecialties within ophthalmology. Key topics include: Optometry; Visual science; Pharmacology and drug therapy in eye diseases; Basic Sciences; Primary and Secondary eye care; Patient Safety and Quality of Care Improvements. This journal is indexed on PubMed

\section{Dovepress}

Central and CAS, and is the official journal of The Society of Clinical Ophthalmology (SCO). The manuscript management system is completely online and includes a very quick and fair peer-review system, which is all easy to use. Visit http://www.dovepress.com/ testimonials.php to read real quotes from published authors. 